not accompanied by the characteristic train of sensory symptoms which we have been considering, and it may be well briefly to refer to two of them. The first is hydromyelia or congenital dilatation of the spinal canal, of which there is a specimen taken from the cord of a child who also bad an occipital meningocele (Fig. 7). It does not give rise to any characteristic symptoms. The second is the formation of cavities in the cord from softening, either from inflammation or vascular obstruction (myélite cavitaire), and of that I show you a specimen taken from the cord of a woman, aged 67 years, who died from hemiplegia due to cerebral thrombosis. No sensory symptoms were detected during life (Fig. 8).

The diagnosis in cases where the symptoms are due to gliosis is usually easy if the sensation has been carefully investigated, because the dissociation of the different forms of temperature is pathognomonic of the condition. The atrophy of the hands, which is an early symptom, differs little from that in cases of progressive muscular atrophy from which the sensory changes at once distinguish it. Neuritis produces a deformity of the hands which closely resembles that caused by syringomyelia, but in neuritic cases common sensation is as a rule affected and electrical examination reveals the reaction of degeneration. There is one disease which causes not only atrophy through neuritis, but also causes similar trophic disturbances, and even the loss of the ends of the digits-viz., leprosy. When syringomyelia was first described it was held by many to be nothing more than leprosy - a view which is still beld by some with reference to the variety which is known as Morvan's disease. The electrical reactions and the absence of dissociation of tactile and painful sensations, together with other positive evidence of leprosy, will usually make the diagnosis clear. No doubt in past years many cases of syringomyelia were unrecognised or labeled "progressive muscular atrophy." In his "Électrisation Localisée" Dachenne of Boulogne figures sections of the cord from a supposed case of this kind which is clearly one of syringomyelia, and another case regarded by Duchenne as one of progressive muscular atrophy was demonstrated upon years afterwards by Charcot as one of syringomyelia. Central hæmorrhage into the cord gives rise to symptoms closely resembling those of syringomyelia but are distinguished by the suddenness of the onset of symptoms. Central gummata are extremely rare but the possibility of their occurrence must be borne in mind. The symptoms would correspond with those of the group gliomatosis, and in all such cases a tentative anti-syphilitic course is prudent.

Prognosis.-In some cases of gliosis as exemplified by the patient before us the disease continues for many years, the severity of the symptoms fluctuating considerably from time to time, but on the whole there is a downward progress, and it is improbable that cases ever recover, the patients usually dying ultimately from some intercurrent malady. In cases of gliomatosis the symptoms develop and increase much more rapidly and terminate fatally, usually through cystitis and septic kidney trouble, in a few years.

Treatment.-No treatment by drugs or other therapentical measures appears to have any effect on the morbid process in the cord. All that can be done is to relieve symptoms on general principles as they occur. Surgical intervention for the purpose of draining the cavity has been recommended but it is doubtful whether it would be effectual and since it has been observed in a number of instances that operations for other purposes have been very badly borne by patients suffering from syringomyelia the risk would probably be greater than the probability of affording relief.

Hospitals and General Practitioners.-A special meeting of the Council of the Charity Organisation Society will be held at the Royal United Service Institution, Whitehall, S.W., on Monday, May 8th, at 4.30 P.M., Sir James Crichton Browne, Bart., M.D. Edin., LL.D., F.R.S., in the chair. The business before the meeting is as follows: 1. Adoption of the second annual report of the general committee for the formation of a cintral hospital board for London. 2. Adoption of a report on general medical practitioners and hospitals. Sir William Broadbent, Bart., M.D. Lond., F.R.S., General the Right Hon. Lord Methuen, K.C.V.O., Dr. W. Knowsley Sibley, and Dr. H. MacnanghtonJones will address the meeting.

\section{ON SOME COMPLICATED CASES OF APPENDICITIS AND THEIR SURGICAL TREATMENT. ${ }^{1}$}

BY H. BETHAM ROBINSON, M.S. LOND., F.R.C.S. ENG., ASSISTANT SURGEON TO, AND SURGEON FOR DISEASES OF THE THROA'T AT', ST. 'THOMAS'S HOSPITAL; SUR(XEON TO THE EAST LONDON HOSPITAL FOR CHILDREN, SHADWELL.

To expound any new facts on such a well-debated subject as appendicitis might seem almost impossible, but the cases which I bring before you this evening, even if well recognised, will, I am sure, prove of interest, not only as illustrating some of the more serious complications of the disease, but as showing how the surgeon is required to be continually on the alert and what measures he may have to adopt to get a successful result. A group of cases I wish particularly to bring before you is where the abscess in relation with the appendix is attended by suppuration later in the neighbourhood of the liver and we may first consider any factors that determine such a sequel. It would be difficult to show whether any particular position of the appendix is more liable to be attended by these complications, especially when we bear in mind how in many cases it is next to impossible to locate its exact direction. The most common site of the appendix is upwards and inwards towards the umbilicus, passing behind the inner margin of the cæcum and ileo-cæcal junction. It is conceivable that suppuration in relation with an appendix thus placed may have more chance of becoming encysted through the early matting together of the folds bounding the ileo-cæcal fossa. If the base of the appendix be the situation of the lesion or the appendix be placed in the iliac fossa or running up on the outer side of the cæcum the encysting of the pus is much more dependent on peritoneal adhesions and matted coils of bowel, as the parieto-colic folds with their ordinary development would not have much limiting influence. It is my experience that the adhesions usually are weaker towards the outer side in the direction of the ascending colon and acting on this it is my special care to very gently handle the parts in this direction. By recalling the anatomy of this region one can imagine (if there are no restraining adhesions and the peritoneum gets fouled on the outer side of the cæcum) how very easy it is for the infection to spread upwards. The recumbent position and the inclined plane on the outer side of the ascending colon leading to the space over the head of the kidney are factors of importance in the accumulation of pus beneath the liver. This intraperitoneal space in the right hypochondrium can contain a large fluid collection and is very readily shut off along its inner side from the general peritoneal cavity. In front are the under surface of the right lobe of the liver and the gall-bladder, both of which are in relation below with the hepatic flexure of the colon; above is the reflection of the peritoneum from the liver and right lateral ligament to the parietal peritoneum over the right kidney and posterior abdominal wall to its outer side; on its inner side are the foramen of Winslow and duodenum; and below is the hepatic flexure of the colon with the commencement of the space along the outer side of the ascending colon. Rutherford Morison ${ }^{2}$ has drawn attention to this space as a seat for fluid collections after gall-bladder operations. It would be expected that a collection here might drain into the lesser sac of the peritoneum, but no doubt this is early sealed off by adhesions, and 1 have determined that this was so in one of my cases, and also it must be borne in mind that no mechanical overflow could take place until late owing to the depth of the fossa posteriorly below the level of the spine, the foramen of Winslow being of course anterior to the spine. The most likely extension from this space would be over the anterior and superior surfaces of the right lobe of the liver round the inferior margin of the gland or round the edge of the right lateral ligament, forming a subdiaphragmatic collection.

With these preliminary anatomical observations I will quote cases which I think illustrate these points.

CASE 1. Gangrene of the appendix with abscess; secondary collection below the right lobe of the liver; operations;

1 A paper read before the Medical Society of London on April 24th, 1899 2 Brit. Med. Jour, vol. ii., 1894, p. 968. 
recovery.-A youth, aged 18 years, was admitted into St. Thomas's Hospital on Jan. 21st, 1898, under the care of $\mathrm{my}$ colleague, Dr. Payne. Having previously always been well the patient had abdominal discomfort on the 19th with loss of appetite, culminating in pain generally distributed at night without nausea. There was little sleep that night but the next morning he seemed better, ate some breakfast, and went to his work; he also took some salts. During the day there were nausea, a good deal of hiccough, and loss of appetite; in the afternoon and night the pain seemed to localise itself to the right iliac fossa causing considerable tenderness. On admission he had a flushed face and furred tongue with a temperature of $102^{\circ} \mathrm{F}$. The abdomen was hard and tense on the right side, especially at the lower part over the iliac fossa where there was considerable tenderness with dulness on percussion. Only the upper part of the abdomen moved during respiration and then only to a slight extent. The abdominal muscles on the right side were contracted so that the last rib and iliac crest were approximated; on palpation a well-marked mass could be detected with special tenderness over McBurney's point. The patient said that on arriving at the hospital he noticed the pain, which had been before in the iliac fossa, shoot up his right side; this lasted for some time and then left an aching pain which soon, however, disappeared. The liver dulness was normal and there was nothing to be detected in the chest. On the 22nd the temperature was $1004^{\circ}$, but the pain was less; there had been constipation for three days. In the afternoon an operation was performed and a collection of pus was found behind the cæcum surrounding the appendix which was directed upwards towards the umbilicus. The appendix was so firmly adherent at its distal end as not to permit of its separation with safety ; its central part, which could be easily freed, was gangrenous on one side. As the whole appendix could not be removed the gangrenous part was incised and a small drainage-tube was inserted and lightly packed round with gauze. The temperature after the operation dropped to normal, but after a few hours began to rise again and he was troubled with slight cough. On the morning of the 24th the temperature was $101.4^{\circ}$, the respirations were 34 , and the pulse was 127. The cough was still troublesome and there was some purulent expectoration. The right side of the chest was dull in front from the fourth rib downwards and from the inferior angle of the scapula behind; over this dull area the breath sounds were diminished and the voice sounds were ægophonic. He was again examined and it was found that a track ran upwards from the wound to the dull area along the ascending colon. An incision was then made just below the costal margin on the right side about four inches long dividing the outer part of the rectus. Thin, purulent, offensive fluid was found occupying a space bounded by the inferior surface of the liver, the front of the kidney, the hepatic flexure of the colon and the duodenum and matted gastro-hepatic omentum on the left. Into this space the probe could be passed up along the ascending colon. There was some fibrinous deposit about the inferior border of the liver creeping round on to the anterior surface. Two tubes were then inserted, one draining the space through the loin and the other tube passing down in the line of the colon to the lower wound. The next morning (the 25th) the temperature was $99^{\circ}$, the pulse was 102 , and the respirations were 32. There was no pain except on coughing and then only in the wound. Through the day and night the temperature rose again, reaching $101.4^{\circ}$ at 8 A.M. on the 26th. His general condition was extremely precarious as he was cyanosed, with bubbling crepitations over both lungs, and the discharge from the wounds was extremely offensive. Fortunately he rallied, the temperature going down during the day, reaching $98^{\circ}$ at midnight and then remained below $100^{\circ}$. The lungs gradually cleared of moist sounds but on the 28th there was some impairment of the percussion ncte with slight friction in the left post-axillary line at the level of the eleventh and twelfth dorsal spines. These disappeared during the next day or two and his further progress was almost uneventful except for a little anxiety owing to the temperature going up on Feb. 7th and remaining so until the 13 th when it finally became normal. This rise was associated with signs of consolidation at the right base. Both drainage tubes were removed on the $11 \mathrm{th}$, but the wounds were a long time closing, the top one being healed on March 17th and the lower one shortly after. wards.
CASE 2. Appendicitis with abscess; secondary collection below the right lobe of the liver; operations; recovery.-A child, aged eight years, was admitted into the East London Hospital for Children, Shadwell, under the care of Dr Donkin, on August 5th, 1898. There was no history of any previous attack. Four days before admission the illness started with vomiting, drowsiness, and fever, and the next day pain was complained of in the right iliac region. All the next day she had the pain and at night the mother gave her castor oil, when she vomited for the second time. On the afternoon of the fourth day she was admitted into the hospital. The bowels had not acted since the commencement of the illness and nothing but milk had been taken. On admission the abdomen hardly moved during respiration and the right hip was flexed. There was very great tenderness and a lump could be felt in the right iliac fossa. Per rectum no lump could be felt bulging from the right side. The temperature was $101^{\circ} \mathrm{F}$. and the pulse was 108 . At midnight the temperature was $102^{\circ}$, but during the 6 th it dropped to $99 \cdot 4^{\circ}$, rising at night to $100 \cdot 8^{\circ}$. On the 7th the patient seemed decidedly better, the temperature being lower, but on the 8 th after rising to $100 \cdot 6^{\circ}$ it fell to normal at midday only to rise again to its original level. The dull area had extended more into the loin and indefinite fluctuation was obtained. On the 9 th the temperature having continued to rise to $102^{\circ} \mathrm{I}$ was asked to see her and as there was undoubtedly pus present immediate operation was advised. The usual skin incision was made and the layers of the abdominal wall were split. On incising the peritonenm a pus cavity was opened which extended up behind the inner side of the cæcum and ileo-creal valve in the line of the normal direction of the appendix. The appendix was embedded in adhesions and no lesion was found in it. The cavity was firmly walled in by adhesions below, but on the outer aspect of the cæcum they were very delicate. The pus was most offensive. A rubber tube was inserted into the cavity and iodoform gauze was lightly packed around it. The temperature was $96^{\circ}$ after the operation and rose again to $100^{\circ}$, gradually coming to normal during the next 24 hours. At midday on the 14th after everything had proceeded satisfactorily the temperature went up to $101^{\circ}$ and remained so with only slight remission up to the afternoon of the 16th when the second exploration was made. On examination on the 16th there was well-defined fulness on the right side of the abdomen below the costal arch and also in the right loin. There was noticed approximation of the last rib and the iliac crest. With respiration there was very little morement on the right side. Dulness extended up from the wound to the costal margin and into the right loin and a fluid thrill could be elicited from front to back. The upper margin of the liver dulness was in the fifth space in the nipple line. From the first wound the discharge was now much thinner and less offensive. As it seemed clear that there was a second collection of pus an incision was made just below and parallel to the costal margin from the linea semilunaris into the right loin. An intraperitoneal pus collection of almost half a pint was opened. This space had for its boundaries the under surface of the liver above, the foramen of Winslow and the duodenum on the inner side, the hepatic flexure of the colon below, and it was completely shut off from the previous collection. There was no extension in front over the anterior surface of the liver. The cavity was washed out with boric lotion and a gauze drain was inserted. The after-effect on the temperature was excellent as it was normal except for a very temporary rise to $100^{\circ}$ on the 21st. On the 19th she had jaundice which perhaps was not surprising considering that the common duct was in the inner wall of the cavity, but this soon disappeared. There is nothing to detail about the further progress of the case. The lower wound was healed at the end of the month but the upper one was not sound until the middle of September, when she went away to the convalescent home.

The above two cases are very much of the same type, each of them having, secondary to the primary lesion, a welldefined collection under the right lobe of the liver and limited to that region. In Case 1 the physical signs in both lungs after the operation did not appear to have any relation. to the inflammation about the liver. From the extension of the liver dulness upwards it was a question before the secondary operation whether the organ was simply pushed up or whether there was as well pleural effusion on this side or a subdiaphragmatic collection. After the operation this upward extension soon disappeared, so it seemed proved that the organ had only been displaced upwards. 
CASE 3. Appendicitis with abscess in the pelvis; secondary inflammation about the liver; subdiaphragmatio abscess (?) ; right empyema; parotid bubo; operations; recovery.-A boy, aged 16 years, was admitted into St. Thomas's Hospital under the care of Dr. Payne on March 15th, 1898. Up to the present illness he had always enjoyed good health. About four weeks before admission he complained of abdominal pain; a fortnight later he had severe vomiting and took to his bed. He was admitted into the hospital with a rigid abdomen, more especially in its lower part, the condition simulating an over-distended bladder. On drawing off the urine no difference was produced in the size of the swelling. Rectal examination proved the existence of fluid in Douglas's pouch. The temperature was $102^{\circ} \mathrm{F}$., the pulse was 100 , and the respirations were 28. In the evening his abdomen was explored by a lateral incision as his symptoms pointed to appendix trouble. The muscular layers were split, not cut, and the peritoneum was opened. Here it was found that matted bowel roofed in the fluid collection and to get at the latter the coils were gently separated and about one pint of pus with some fæcal matter was set free. The appendix was not found. A glass tube was put into the pelvis and a gauze drain into the upper part of the wound. His progress for a week was fairly satisfactory and then his temperature began to go up, reaching from $102^{\circ}$ to $103^{\circ}$. On March 22nd two small rubber tubes were substituted for the previous drains as the discharge had considerably lessened. On the 23 rd he was noticed to be developing a parotid bubo on the left side which remained for some time without showing any signs of softening, but eventually an abscess formed and it was opened on April 6th. A large piece of necrosed tissue, apparently lymph, came away on the 2 nd from the abdominal wound followed by a considerable quantity of foul-smelling pus. There was marked diminution in the extent of the dull area and of abdominal tenderness. On the 7th he had severe pain on the left side below the cardiac area and friction could be heard along the line of the seventh rib into the axilla. There was some dulness behind from the angle of the scapula downwards. On the 8th he complained of pain in the abdomen and on coughing brought up several ounces of thin muco-purulent sputum with very offensive odour. On examination on the 9th the abdomen was not painful or tender and moved fairly well and equally on respiration. The impaired resonance remained the same on the left side without evidence of fluid collecting. The parotid bubo had discharged freely and the temperature had gone down. There was complaint of pain in the right shoulder. The temperature came down to normal on the 11th, but the lung-signs on the left side remained; if anything, the breath-sounds were less marked. On the 13th the temperature went up again to $102.8^{\circ}$ and the next morning there were signs of fluid in the right pleura with friction in the axilla and diminished breath and roice sounds at the base in front and behind. The liver dulness began at the fifth rib in the nipple line and at the sixth in the mid-axilla. The upper part of the abdomen was explored on the 16th by an incision parallel with the right costal margin starting from the linea semilunaris. Dense adhesions were found on the upper and under surfaces of the liver, but no pus was discovered. A hypodermic needle was passed into the pleura and a negative result was obtained. On the 27 th the physical signs at both bases continued as before except that now there was no friction on the right side. He was cheerful, had no pain, slept well and took his food readily, but the temperature was still irregular. On May 6th there was some return of abdominal pain, but no lesion was detected to explain this. The parotid wound had almost healed. The upper abdominal wound had closed but the lower one still discharged. On the 16th the physical signs in the lungs were the same, but the persistence of the nightly rise of temperature strongly suggested pus. It was determined to explore the right chest again on the 25th as some cedema was noticed over the lower spaces laterally. An incision was made over the right tenth rib in the posterior part of the axillary line and about two inches of bone were removed. On opening the pleura very foul pus was evacuated from a localised cavity apparently pleural this was washed out with boric acid and as there was considerable hæmorrhage the space had to be plugged with cyanide gauze for some hours. After the operation the temperature fell to $96.4^{\circ}$ and then rose again to just over the normal line. At this period the patient was in a terribly emaciated state, weighing only 4 st. $6 \mathrm{lb}$. From
this time on he rapidly improved, his temperature remaining normal excepting for a sudden unexplained rise to $103 \cdot 6^{\circ}$ on June $3 r d$. He took his food well and gained flesh reaching 5 st. at the end of the month, by which time his wound had healed. He was discharged from the hospital on July 10th.

The appendix abscess in this case appears to have gradually collected in the pelvis and to have been roofed in by matted small intestine. As to the position of the appendix I cannot say for certain, but there is a strong probability that it. direction was downwards and inwards towards the pelvis and possibly overhanging the pelvic brim. As in the former cases, there was spreading of peritoneal infection along the colon to the liver as was shown by the extensive adhesions on exploration. There was no actual proof of any subdiaphragmatic collection, but the lung signs terminating in the basal empyema on the right side gave some colouring to this belief. The association of the parotid bubo may be noted as it has been so frequently in relation with peritoneal inflammation especially when in the pelvis.

The next case is one of a different type and of considerable interest from the subacute nature of the symptoms and from the local conditions found on exploration.

CASE 4. Appendicitis; secondary abscess below the costal margin superficially placed ; facal fistula; recovery.-A boy, aged 12 years, was admitted into St. Thomas's Hospital under the care of my colleague, Dr. Payne, on April 13th 1898. He had always had good health and so this seemed undoubtedly his first attack. On April 7th he was seized with severe pain in the abdomen and the next day he commenced to vomit which continued up to the date of admission. During these days practically no nourishment was taken. The bowels were last opened on April 6th. On admission he was found to be well nourished but pale. The abdomen was rather distended and did not move well on respiration. It was resonant all over except in the right iliac fossa where a distinct tumour could be felt. Over this area it was extremely tender, especially over McBurney's point. The rest of the abdomen was not tender on palpation, the liver dulness was normal, and the spleen was not felt. His temperature was normal and the pulse was 90. During the next few days there was gradual loss of tenderness with reduction in size of the inflammatory mass. He vomited once or twice but was able to take fluid nourishment. His bowels were opened naturally on the 18th. His temperature reached $100^{\circ} \mathrm{F}$. on the three successive evenings of the 14th, 15th, and 16th, but was otherwise normal. On the evening of the 22 nd after having a more substantial diet he had a severe attack of vomiting. On examination a hard lump of about the size of a walnut could be felt at the outer edge of the right rectus about one inch below the costal margin; this seemed in the abdominal wall; it did not move with respiration and was tender on pressure. Nothing could now be felt in the iliac fossa, but there was a cordlike induration starting at about the lower quarter of the rectus running upwards along its outer margin to the lump above. On the 25th I operated, making an incision over the upper lump parallel to the costal margin; beneath the transversalis a little pocket of very foetid pus was opened. An incision was then made below over McBurney's point and the lower end of the cord-like thickening was exposed and again a little pus was set free. A probe was passed into the lower end of what proved to be a sinus lined with soft granulations which led up to the small abscess. Downwards the probe went towards the pelvis and this track was opened up by dividing some of the outer fibres of the rectus and the deep epigastric artery. No more pus was discovered. The probe could be easily felt in the pelvis per rectum in relation with its right wall, but it was difficult from this site and from what was found in the wound to say whether the granulation track was in the subperitoneal layer or among peritoneal adhesions. No bowel was displayed. A drainage tube was put in the lower track and a gauze drain in the upper one; the wound was partially sutured. His condition after this for some days was satisfactory, but then his temperature began to go up at night, reaching its maximum $\left(102^{2} 2^{\circ}\right)$ on the 7 th, on which day there was a frecal discharge from the wound and after that a gradual reduction in temperature. On the 14th the lower sinus was again explored when at the bottom an opening was found in the bowel armitting the tip of the finger; all round the opening was granulation tissue and it
was difficult to say whether the bowel so perforated was the 
base of a dilated appendix or the cæcum, but I thought the latter. From the rotten state of the bowel wall it was impossible to close the opening without doing an extenşive operation and so it was not attempted. His upper wounds soon healed and the fæcal fistula gradually contracted but yet allowed considerable discharge when he went to the convalescent home on July 1st. He came into the hospital again in December, his fistula having contracted up to a very small opening; as there was only a slight fæcal discharge which could be easily controlled I determined to wait events knowing how unsatisfactory attempts to close these fistulæ are. Since then I have seen nothing more of him.

The main feature of interest in this case was the possible unusual sequence of events after the primary lesion. On exploration it was extremely difficult to say whether the pus collection outside the margin of the rectus was in the subperitoneal tissue or in the peritoneal cavity. If in the subperitoneal tissue it must be assumed that the peritoneum had become adherent about the base of the appendix or cæcum and then given way, leading to the gradual travelling up of the pus towards the costal margin. If the lesion was intraperitoneal we have the same course of events as in the previous cases, only on a smaller and less active scale. The infection spread up on the anterior surface or outer margin of the colon, the latter becoming adherent to the abdominal wall. Now an apparent difficulty, the abscess just below the costal margin, is not so, for if the lower edge of the liver were adherent to the colon and both of them to the parietal peritoneum at the costal margin the pus might easily make its way forwards. The ease with which the probe in the bottom of the wound could be felt per rectum also gives some support to the intraperitoneal site, for it would pass among adhesions into Douglas's pouch, not an uncommon direction for pus to take, whereas if this track had been in the subperitoneal tissue the probe would have seemed more distant.

To summarise, the following points may be deduced from this paper : (1) in a small proportion of cases of appendicitis with abscess there is intraperitoneal extension to the liver region; (2) in relation with the liver pus may be only below it or also above it "subdiaphragmatic"; and (3) clinically there is considerable difficulty in determining when the apparent upper border of the liver is raised, how much of this is due to simple displacement upwards by a fluid collection or to a subdiaphragmatic abscess or to an associated lesion at the lung bases.

Upper Wimpole-street $\mathbf{W}$.

\section{THE SERUM TREATMENT OF PLAGUE.}

\section{By FRANK G. CLEMOW, M.D. Edin., D.P.H. Cantab.}

WHLLE the use of an organic fluid obtained directly or indirectly from the bacillus of plague has already led to most successful and even brilliant results as a prophylactic in this disease, it must be regretfully admitted that the trials made of similar fluids as curatives have hitherto met only with failure or with the most partial success.

Careful experiments have now been made with, so far as I am aware, four different fluids of this kind in the treatment of plague. They are associated with the names of the following observers: M. Haffkine, M. Yersin, Professor Lustig, and M. Roux. That associated with the name of M. Hafflkine is now being widely and successfully employed in India as a prophylactic, but it was originally investigated as a curative agent. It will be remembered that the conclusion which was come to as to its value in this respect was that it possessed no curative powers and that its effect, if anything, was rather detrimental than otherwise. It was consequently abandoned almost immediately as a curative agent and I am not aware that any further attempt has been made to search for a cure among fluids prepared in this or in a similar manner. M. Haffkine's fluid, it is almost superfluous to state, is a pure growth of the plague bacillus in which the organisms themselves have been killed by raising the growth to a temperature of $58^{\circ} \mathrm{C}$. for one hour. It contains the dead bodies of the killed bacilli and the chemical products of their growth which are apparently (so far, at least, as their prophylactic powers are concerned) uninjured by the exposure to this temperature. The other three "curative" agents are all derived from the blood serum of a horse which has been modified by repeated injections into the veins of the animal of a culture of plague bacilli.

The first serum of this kind to be experimented with was one prepared by M. Yersin in 1896 and the results of its administration in a few cases of plague in Amoy led, it will be remembered, to very sanguine hopes as to its future. ${ }^{1}$ Unfortunately these hopes were not borne out by further trials in Bombay and elsewhere and my own experience, which is given in detail below, has led me to the conclusion that this serum even in comparatively large doses is entirely without effect upon the symptoms of plague.

A second serum is that prepared by Professor Lustig of Florence. Experiments prolonged over two years have now been made with this serum in Bombay, partly by Professor Lustig himself and partly by his assistants Dr. Galeotti and Dr. Polverini. It has been claimed for this serum that it reduces the case-mortality of plague from 86 per cent. to 40 per cent. $^{2}$

The third serum is that prepared by M. Roux in the Pasteur Institute. The most extensive trial of this serum has been made by M. Simond who has charge of the French colonial bacteriological station at Saigon and who recently visited India. M. Simond's results have not, so far as I am aware yet been published, but he informed me when in Calcutta last September that he had obtained very promising results with the serum, the mortality being reduced by about onehalf.

Of these serums and their effect in the treatment of plague I have had personal experience of two only-M. Yersin's and Professor Lustig's. The number of cases which I have seen treated by the latter is perhaps too small to base a final conclusion on their results, but in the case of the former I have been in a position to observe and to take part in a care fully conducted trial in which 50 cases were submitted to this form of treatment and 50 alternate cases noted as controls, and the results of this trial have enabled me to form a very definite, though unfortunately unfavourable, opinion as to the value of this particular serum.

50 Cases of Plague treated with M. Yersin's Serum.

During the months of March and April of last year 50 patients suffering from plague were submitted to the serum treatment in the wards of the old Government House Hospital, of which I was then in charge. The serum in question was prepared in the Imperial Institute of Experimental Medicine in St. Petersburg and was brought to India by the Russian Plague Commission, of which Dr. Yassenski was the principal member. The injections were for the greater part administered by Dr. Yassenski.

Mode of preparation of the serum.-A peptone-agar culture of the plague bacillus, prepared under special precautions to obtain an even growth, is rubbed up after 24 hours' growth with four or five cubic centimetres of a 0.5 per cent. solution of salt. A smooth emulsion should result. This is exposed in a water bath to a temperature of $58^{\circ} \mathrm{C}$. for one hour. The emulsion is injected into the veins of a horse and reaction usually follows. The degree of reaction varies very considerably in different horses. A second injection is given 10 days after complete cessation of the reaction from the first. The serum obtained from the horse's blood is tested before use upon white mice, 20 grammes in weight. The serum is diluted with 0.5 per cent. salt solution and one mouse receives one-fifth of a cubic centimetre, a second one-tenth and a third one-twentieth. Twelve hours later each of these mice and a control one receive an injection of plague virus sufficient to kill the control mouse in two days. If the mouse which received one-tenth of a cubic centimetre of the serum remains alive the serum is fit for use on human beings. Before storing the serum is diluted with 0.5 per cent. of carbolic acid.

Mode of storage and transport.-The serum is stored in small glass flasks drawn out to a long neck. Each flask holds 10 cubic centimetres of the serum. The neck is sealed in the blow-flame and no cork or sealing-wax is required. The method appears to me to be preferable to any that I have yet seen for storing organic fluids. The serum prepared in the Pasteur Institute in Paris is put up in small bottles closed with corks and sealing wax, and a simular method is employed for the storage of M. Haffkine's prophylactic. The latter has the still further disadvantage that the fluid is

1 Annales de l'Institut Pasteur, January, 1897, pp. 81-93. 